\title{
Scattering Theory for a Stratified Acoustic Strip with Short- or Long-Range Perturbations
}

\author{
By
}

Elizabeth CRoC* and Viorel IfTIMIE**

\begin{abstract}
We consider the acoustic propagator $H=-\nabla \cdot \rho \nabla$ acting in $L^{2}(\Omega)$ with $\Omega:=$ $\Omega^{\prime} \times \mathbb{R}$ and $\Omega^{\prime}$ a bounded open set in $\mathbb{R}^{n-1}, n \geq 2$. The real-valued function $\rho$ belongs to $L^{\infty}(\Omega)$, and is bounded from below by $c>0$. We assume there exist two strictly positive constants $c_{1}$ and $c_{2}$ and two perturbations, $\delta^{S}$ of short-range type and $\delta^{L}$ of long-range type, such that $\rho=c_{j}+\delta^{S}+\delta^{L}$ on $\Omega_{j}:=\left\{\left(x^{\prime}, x_{n}\right) \in \Omega \mid(-1)^{j} x_{n}>\right.$ $0\}, j=1,2$. We build two modified free evolutions $U_{j}(t), j=1,2$, such that the wave operators $\Omega_{j}^{ \pm}:=s-\lim _{t \rightarrow \pm \infty} e^{i t H} U_{j}(t), j=1,2$, exist and are asymptotically complete.
\end{abstract}

\section{Contents}

$\S 1$. Introduction and Results

$\S 2 . \quad$ Spectral Preliminaries

$\S 3 . \quad$ Eikonal Equation

$\S 4$. A Class of Fourier Integral Operators

$\S 5$. Identification Operators

$\S 6$. Existence of the Generalized Wave Operators

$\S 7 . \quad$ Asymptotic Completeness

$\S 8$. Free Modified Evolutions

References

Communicated by T. Kawai. Received January 24, 2001.

2000 Mathematics Subject Classification(s): 35P25.

*Université de Provence, CMI, UMR CNRS 6632, 39, rue F. Joliot-Curie, 13453 Marseille Cedex 13, France.

**Université de Bucarest, Faculté de Mathématiques, 14 rue Academiei, Bucarest, Roumanie. 


\section{$\S 1 . \quad$ Introduction and Results}

Let $\Omega^{\prime} \subset \mathbb{R}^{n-1}, n \geq 2$ be a Lipschitz bounded open set (or according to Stein [13] a domain with a "boundary with minimal regularity"). Then the cylinder $\Omega:=\Omega^{\prime} \times \mathbb{R}$ is also a Lipschitz domain and the Sobolev spaces $\mathcal{H}^{s}(\Omega)$ and $\mathcal{H}_{0}^{s}(\Omega), s \in \mathbb{R}$, have the usual properties.

We consider a function $\rho: \Omega \mapsto \mathbb{R}_{+}^{*}$ satisfying the following assumptions:

(i) $\rho$ and $1 / \rho$ belong to $L^{\infty}(\Omega)$.

(ii) There exist two strictly positive constants $c_{1}, c_{2}$ and two real-valued functions $\delta^{S}, \delta^{L}: \Omega \mapsto \mathbb{R}$, such that:

a) $\rho=c_{j}+\delta^{S}+\delta^{L}$ on $\Omega_{j}:=\left\{x=\left(x^{\prime}, x_{n}\right) \in \Omega /(-1)^{j} x_{n}>0\right\}, j=1,2$.

b) $\delta^{S} \in L^{\infty}(\Omega)$ is a short-range perturbation, i.e. there exist constants $C>0$ and $\theta \in(0,1]$ such that

$$
\left|\delta^{S}(x)\right| \leq C\langle x\rangle^{-1-\theta} \quad \text { a.e. (almost everywhere) on } \Omega \text {, }
$$

where $\langle x\rangle:=\left(1+|x|^{2}\right)^{1 / 2}$ and $|x|$ is the Euclidean norm of $x \in \mathbb{R}^{n}$.

c) $\delta^{L} \in C^{\infty}(\bar{\Omega})$ is a long range perturbation, i.e. there exists a constant $\theta \in(0,1]$ such that

$$
\forall \alpha \in \mathbb{N}^{n}, \exists C_{\alpha}>0,\left|\partial^{\alpha} \delta^{L}(x)\right| \leq C_{\alpha}\langle x\rangle^{-\theta-|\alpha|} \text { on } \Omega
$$

Remark 1.1. a) We can assume that $\delta^{L}$ depends only on $x_{n}$. Indeed, according to [11], each connect component of $\Omega^{\prime}$ is a finte union of open sets starred with respect to a ball. Therefore by applying conveniently Taylor's formula with respect to $x^{\prime}$, we get $\delta^{L}(x)=\delta_{0}^{L}\left(x_{n}\right)+r^{S}(x)$ where $r^{S}$ is shortrange perturbation and $\delta_{0}^{L}$ is a long-range perturbation.

b) We can also assume with a modification of $\delta^{S}$ that

$$
\left|\delta^{L}(x)\right| \leq 1 / 2 \min \left\{c_{1}, c_{2}\right\}, x \in \Omega .
$$

The quadratic form $h$, with domain $D(h):=\mathcal{H}_{0}^{1}(\Omega)$, defined by

$$
h(u, v):=\int_{\Omega} \rho \nabla u \cdot \nabla \bar{v} d x, \quad u, v \in \mathcal{H}_{0}^{1}(\Omega),
$$

is symmetric, non negative and closed. Kato's representation theorem (Chapter VI of [10]) gives a unique self-adjoint operator in $L^{2}(\Omega)$ with domain

$$
\begin{aligned}
D(H) & =\left\{u \in \mathcal{H}_{0}^{1}(\Omega) /-\nabla \cdot \rho \nabla u \in L^{2}(\Omega)\right\}, \\
H u & =-\nabla \cdot \rho \nabla u \quad \text { if } u \in D(H) .
\end{aligned}
$$


The spectral theory of the operator $H$, and a limiting absorption principle (under more general assumptions), have been studied in [7] (refer also to [4] at the origin of these works). When the perturbation $\delta^{L}$ is zero (and $c_{j}, j=1,2$, not necessarily constants), the scattering theory for $H$ has been made in [5] by comparing $H$ to the "free" operators $H_{j}, j=1,2$, defined analogously to $H$ by substituting $c_{j}$ for $\rho$ and by using some aspects in relation with a 3-body Hamiltonian of quantum mechanics.

In our case where the functions $c_{j}, j=1,2$, are constant, the "free" operators $H_{j}, j=1,2$, are the self-adjoint operators in $L^{2}(\Omega)$ defined by

$$
\begin{aligned}
D\left(H_{j}\right) & =\left\{u \in \mathcal{H}_{0}^{1}(\Omega) /-\Delta u \in L^{2}(\Omega)\right\}, \\
H_{j} u & =-c_{j} \Delta u \text { if } u \in D\left(H_{j}\right) .
\end{aligned}
$$

Note that $D\left(H_{j}\right)=\mathcal{H}_{0}^{1}(\Omega) \cap \mathcal{H}^{2}(\Omega)$ if $\Omega^{\prime}$ would have the exterior ball property (see $[2])$.

In the case of a long-range perturbation, it is no longer possible to compare the evolution $e^{-i t H}$ with the free evolutions $e^{-i t H_{j}}, j=1,2$, and we have to find modified free evolutions $U_{j}(t), j=1,2$. There exist a lot of results concerning the scattering theory for stratified media with short-range perturbations (see for instance [3], [6], [14]). As far as we know, there is no result for such media with long-range perturbations.

The main result of this paper is the following theorem:

Theorem 1.1 (Existence and completeness of the modified wave operators). There exist modified free evolutions $\left\{U_{j}(t)\right\}_{t \in \mathbb{R}}, j=1,2$, where $U_{j}(t)$ are bounded operators in $L^{2}(\Omega)$ determined by $c_{j}, \Omega^{\prime}$ and $\delta^{L}$, such that the modified wave operators

$$
\Omega_{j}^{ \pm}:=s-\lim _{t \mapsto \pm \infty} e^{i t H} U_{j}(t)
$$

exist and are asymptotically complete, i.e.

$$
\mathcal{H}_{a c}(H):=\bigoplus_{j=1,2} \operatorname{Ran} \Omega_{j}^{ \pm}
$$

where $\mathcal{H}_{a c}(H)$ is the subspace of absolute continuity of the operator $H$.

Let $\mathcal{F}_{n}$ be the partial Fourier transform on $L^{2}(\Omega)$ defined for $u \in C_{0}^{\infty}(\Omega)$ and $\left(x^{\prime}, \xi\right) \in \Omega^{\prime} \times \mathbb{R}$ by

$$
\mathcal{F}_{n} u\left(x^{\prime}, \xi\right):=(2 \pi)^{-1 / 2} \int_{\mathbb{R}} u\left(x^{\prime}, x_{n}\right) e^{-i x_{n} \xi} d x_{n} .
$$


We set $\mathcal{H}=L^{2}(\Omega), \mathcal{H}^{ \pm}:=\left\{u \in \mathcal{H} /\left(\mathcal{F}_{x_{n}} f\right) u\left(x^{\prime}, \xi_{n}\right)=0\right.$ if $\left.x^{\prime} \in \Omega^{\prime}, \mp \xi_{n}>0\right\}$ and $\mathcal{H}_{a c, j}^{ \pm}:=\operatorname{Ran} \Omega_{j}^{ \pm}$. Then we have the following corollary:

Corollary 1.1. 1) One has $\operatorname{Ker} \Omega_{1}^{ \pm}=\operatorname{Ker} \Omega_{2}^{\mp}=\mathcal{H}^{ \pm}$.

2) The operators $\Omega_{1}^{ \pm}: \mathcal{H}^{\mp} \rightarrow \mathcal{H}_{a c, 1}^{ \pm}$and $\Omega_{2}^{ \pm}: \mathcal{H}^{ \pm} \rightarrow \mathcal{H}_{a c, 2}^{ \pm}$are unitary.

3) Setting $\Omega^{ \pm}:=\Omega_{1}^{ \pm} \oplus \Omega_{2}^{ \pm}$, the scattering operator $S:=\left(\Omega^{+}\right)^{*} \Omega^{-}$is unitary from $\mathcal{H}=\mathcal{H}^{+} \oplus \mathcal{H}^{-}$onto $\mathcal{H}^{-} \oplus \mathcal{H}^{+}=\mathcal{H}$.

For the proofs, we use some results of [7], in particular the limiting absorption principle, and an idea of Isozaki and Kitada [9] improved by Yafaev [17]. These authors have built wave operators for the Schrödinger operator with an identification $\mathcal{J}$, defined as a Fourier integral operator and allowing to prove the asymptotic completeness. The modified free evolutions follow by the stationary phase method. Nevertheless, the existence of thresholds gives some problems.

The paper is organized as follows. In Section 2, we collect from [4] and [7] the needed results concerning the operators $H$ and $H_{j}$. In Section 3 , we study the eikonal equation. It defines the phase function of a Fourier integral operator on $\mathbb{R}$ considered in Section 4 . The operators of identification are built in Section 5. The existence of the generalized wave operators is proved in Section 6, and their completeness in Section 7. Finally, the construction of the free evolutions $U_{j}(t)$ and the proofs of Theorem 1.1 and Corollary 1.1 are given in Section 8.

Let us give some notations. The norm (respectively the scalar product) in a normed (respectively Hilbert) space $E$ is denoted by $\|\cdot\|_{E}$ (respectively $\left.(\cdot, \cdot)_{E}\right)$. The space of bounded (respectively compact) linear operators from a Banach space $E$ to a Banach space $F$ equipped with the uniform operator topology is denoted by $\mathcal{B}(E, F)$ and by $\mathcal{B}(E)$ if $E=F$ (respectively $\mathcal{K}(E, F)$ and by $\mathcal{K}(E)$ if $E=F)$. If $H$ is a self-adjoint operator in a Hilbert complex space, the spectrum of $H$, (respectively the essential spectrum, the singular continuous spectrum, the absolutely continuous spectrum, the set of eigenvalues, the orthogonal projection on the subspace of absolute continuity $\mathcal{H}_{a c}(H)$ ) are denoted by $\sigma(H)$ (respectively $\left.\sigma_{e s s}(H), \sigma_{s c}(H), \sigma_{a c}(H), \sigma_{p}(H), P_{a c}(H)\right)$.

\section{$\S 2 . \quad$ Spectral Preliminaries}

We identify the space $L^{2}(\Omega)$ with the direct integral $\int_{\mathbb{R}}^{\oplus} L^{2}\left(\Omega^{\prime}\right) d \xi$, so that $\mathcal{F}_{n}$, the partial Fourier transform (1.9), is unitary. We set $\widehat{H}_{j}:=\mathcal{F}_{n} H_{j} \mathcal{F}_{n}^{*}=$ 
$\int_{\mathbb{R}}^{\oplus} \widehat{H}_{j}(\xi)$, with $\widehat{H}_{j}(\xi), \xi \in \mathbb{R}$, the self-adjoint operator on $L^{2}\left(\Omega^{\prime}\right)$ defined by

$$
\begin{aligned}
D\left(\widehat{H}_{j}(\xi)\right) & =\left\{v \in \mathcal{H}_{0}^{1}\left(\Omega^{\prime}\right) / \Delta^{\prime} v \in L^{2}\left(\Omega^{\prime}\right)\right\}, \\
\widehat{H}_{j}(\xi) v & =-c_{j} \Delta^{\prime} v+c_{j} \xi^{2} v \quad \text { if } v \in D\left(\widehat{H}_{j}(\xi)\right),
\end{aligned}
$$

where $\Delta^{\prime}$ is the Laplace operator on $\Omega^{\prime}$. The operator $\widehat{H}_{j}(\xi)$ is with a compact resolvent, and if $\left(\lambda_{k}\right)_{k \geq 1}, 0<\lambda_{1} \leq \lambda_{2} \leq \ldots$, is the sequence of eigenvalues of the Dirichlet problem for $-\Delta^{\prime}$ on $\Omega^{\prime}$ and $\left(V_{k}\right)_{k \geq 1}$ is an orthonormal basis of $L^{2}\left(\Omega^{\prime}\right)$, elements of which are real-valued eigenfunctions of the same problem associated to the above eigenvalues $\left(\lambda_{k}\right)_{k \geq 1}$, then the $V_{k}, k \geq 1$ are also eigenfunctions of $\widehat{H}_{j}(\xi)$ associated to the eigenvalues

$$
\lambda_{j k}:=c_{j}\left(\lambda_{k}+\xi^{2}\right), \quad j=1,2, k \geq 1 .
$$

Note that $V_{k} \in C^{\infty}(\Omega)$.

It is known (see [7]) that $H_{j}$ is purely absolutely continuous and that $\sigma\left(H_{j}\right)=\left[c_{j} \lambda_{1},+\infty\right), j=1,2$. The generalized eigenfunctions of $H_{j}$ are independent of $j=1,2$, and defined by

$$
\Phi_{k}(x, \xi):=(2 \pi)^{-1 / 2} e^{i x_{n} \xi} V_{k}\left(x^{\prime}\right), \quad x=\left(x^{\prime}, x_{n}\right) \in \Omega, \xi \in \mathbb{R}, k \geq 1 .
$$

For $f \in L^{2}(\Omega)$ and $k \geq 1$, we set

$$
\begin{aligned}
f_{k}\left(x_{n}\right) & :=\int_{\Omega^{\prime}} f\left(x^{\prime}, x_{n}\right) V_{k}\left(x^{\prime}\right) d x^{\prime}, x_{n} \in \mathbb{R} \\
\widehat{f}_{k}(\xi) & :=(2 \pi)^{-1 / 2} \int_{\mathbb{R}} e^{-i x_{n} \xi} f_{k}\left(x_{n}\right) d x_{n}=\int_{\Omega} f(x) \overline{\Phi_{k}(x, \xi)} d x, \xi \in \mathbb{R},
\end{aligned}
$$

where both last integrals converge in $L^{2}(\mathbb{R})$.

The following properties can be found in [15], [4] and [7].

Proposition 2.1. 1) For $x \in \Omega, j=1,2, k \geq 1, \xi \in \mathbb{R}$,

$$
-c_{j} \Delta_{x} \Phi_{k}(x, \xi)=\lambda_{j, k}(\xi) \Phi_{k}(x, \xi) .
$$

2) For $f \in L^{2}(\Omega)$,

$$
f(x)=\sum_{k \geq 1} \int_{\mathbb{R}} \widehat{f}_{k}(\xi) \Phi_{k}(x, \xi) d \xi
$$

where the series converges in $L^{2}(\Omega)$.

3) For $f, g \in L^{2}(\Omega)$,

$$
(f, g)_{L^{2}(\Omega)}=\sum_{k \geq 1} \int_{\mathbb{R}} \widehat{f}_{k}(\xi) \overline{\widehat{g}_{k}(\xi)} d \xi .
$$


4) For $f \in D\left(H_{j}\right)$,

$$
{\widehat{\left(H_{j} f\right)_{k}}}_{k}(\xi)=\lambda_{j k}(\xi) \widehat{f}_{k}(\xi) .
$$

5) For $f \in L^{2}(\Omega)$ and $\varphi$ a bounded Borel function on $\mathbb{R}$,

$$
\left(\widehat{\varphi\left(H_{j}\right) f}\right)_{k}(\xi)=\varphi\left(\lambda_{j, k}(\xi)\right) \widehat{f}_{k}(\xi) .
$$

6) The operator $\mathcal{F}: L^{2}(\Omega) \rightarrow \int_{\mathbb{R}}^{\oplus} L^{2}\left(\Omega^{\prime}\right)$ defined for $f \in L^{2}(\Omega)$ by

$$
(\mathcal{F} f)(\xi):=\sum_{k \geq 1} \widehat{f}_{k}(\xi) V_{k}
$$

is unitary.

Proposition 2.2 (see Theorem 0.2 of [7]).

1) $\inf \sigma(H)>0$.

2) $\sigma_{\text {ess }}(H)=[\mu,+\infty)$, with $\mu:=\min \left\{c_{1} \lambda_{1}, c_{2} \lambda_{2}\right\}$.

3) $\sigma_{s c}(H)=\emptyset$.

4) Let $\tau(H):=\left\{c_{j} \lambda_{k} / j=1,2, k \geq 1\right\}$ be the set of thresholds of $H$, then $\sigma_{p}(H) \cup \tau(H)$ is closed and countable. The elements of $\sigma_{p}(H) \backslash \tau(H)$ are eigenvalues of finite multiplicity that can accumulate at the thresholds (or at infinity) only.

To formulate the limiting absorption principle for $H$ (see Theorem 0.3 of $[7])$, we need the weighted Sobolev spaces $\mathcal{H}_{(t)}^{s}(\Omega):=\left\{u \in \mathcal{D}^{\prime}(\Omega) /\langle\cdot\rangle^{t} u \in\right.$ $\left.\mathcal{H}^{s}(\Omega)\right\}, t$ and $s$ real numbers. These spaces are equipped with the natural norms $\left\|\langle\cdot\rangle^{t} u\right\|_{\mathcal{H}^{s}(\Omega)}$. When $t \geq 0$, the injections $\mathcal{H}_{(t)}^{-1}(\Omega) \hookrightarrow \mathcal{H}^{-1}(\Omega)$ and $\mathcal{H}_{0}^{1}(\Omega) \hookrightarrow \mathcal{H}_{(-t)}^{1}(\Omega)$ are continuous.

Proposition 2.3. Let $t>1 / 2$ be a real number. Then for each compact $K$ included in $\mathbb{R} \backslash\left(\sigma_{p}(H) \cup \tau(H)\right)$, one has

$$
\sup _{\lambda \in K, 0<\varepsilon<1}\left\|(H-\lambda-i \varepsilon)^{-1}\right\|_{\mathcal{B}\left(\mathcal{H}_{(t)}^{-1}(\Omega), \mathcal{H}_{(-t)}^{1}(\Omega)\right)}<+\infty .
$$

Finally, we need the following lemma.

Lemma 2.1 (see Proposition 5.7 of $[7]$ ). Let $\alpha_{1}, \alpha_{2} \in C^{\infty}(\Omega)$ be two functions depending only on $x_{n}$, such that $\alpha_{1}=0$ when $x_{n}$ is large enough, $\alpha_{2}=0$ when $\left(-x_{n}\right)$ is large enough, and $\alpha_{1}+\alpha_{2}=1$ when $\left|x_{n}\right|$ is large enough. Then for every $\beta \in C_{0}^{\infty}(\mathbb{R})$, the operator $\beta(H)-\sum_{j=1,2} \alpha_{j} \beta\left(H_{j}\right)$ belongs to $\mathcal{K}\left(L^{2}(\Omega)\right)$. 


\section{$\S 3 . \quad$ Eikonal Equation}

We fix $\Lambda:=[a, b]$ a compact interval in $\mathbb{R} \backslash\left(\tau(H) \cup \sigma_{p}(H)\right)$ and $\beta \in C_{0}^{\infty}(\mathbb{R})$ a real-valued function equal to 1 on $\Lambda$ such that $\operatorname{supp} \beta$ (the support of $\beta$ ) is an interval $\Lambda_{0}:=\left[a_{0}, b_{0}\right]$ also in $\mathbb{R} \backslash\left(\tau(H) \cup \sigma_{p}(H)\right)$.

For $j=1,2$, there exists a unique $k_{j}(\Lambda) \in \mathbb{N}^{*}$ such that $c_{j} \lambda_{k_{j}(\Lambda)}<a<$ $b<c_{j} \lambda_{k_{j}(\Lambda)+1}$. We define the real-valued function $\beta_{j k} \in C_{0}^{\infty}(\mathbb{R})$ by

$$
\beta_{j k}(\xi):=\beta\left(\lambda_{j k}(\xi)\right), \quad \xi \in \mathbb{R}, j=1,2, k \geq 1 .
$$

We have $\beta_{j k}=0$ if $k \geq k_{j}(\Lambda)+1$ and there exists $\varepsilon_{j}(\Lambda)>0$ such that $\beta_{j k}(\xi)=0$ if $|\xi| \leq \varepsilon_{j}(\Lambda)$ and $k \leq k_{j}(\Lambda)$.

For $j=1,2, k \geq 1$, let $\alpha_{j k} \in C^{\infty}(\mathbb{R})$ be a real-valued function such that

$$
\alpha_{j k}\left(x_{n}\right)=1 \text { if }(-1)^{j} x_{n} \geq 2 R_{k}, \alpha_{j k}\left(x_{n}\right)=0 \text { if }(-1)^{j} x_{n} \leq R_{k}, x_{n} \in \mathbb{R}
$$

where $R_{k}$ will be chosen large enough.

Finally, we define the real-valued function $\gamma_{j k} \in C^{\infty}\left(\mathbb{R}^{2}\right)$ by

$$
\gamma_{j k}\left(x_{n}, \xi\right)=: \alpha_{j k}\left(x_{n}\right) \beta_{j k}(\xi), \quad x_{n}, \xi \in \mathbb{R}, j=1,2, k \geq 1 .
$$

We can assume $\theta<1$ in (1.2). Then we have the following proposition:

Proposition 3.1. For every $j=1,2$ and $k \geq 1$, there exist functions $\psi_{j k}$ and $r_{j k}$ in $C^{\infty}\left(\mathbb{R} \times \mathbb{R}^{*}\right)$, with the following properties:

1) $\psi_{j k}$ is real-valued and if $m \in \mathbb{N}^{*}$ and $m \theta>1$, for all $p, q \in \mathbb{N}$, one has, with constants $C_{p q}$ depending also on $j$ and $k$, the inequalities:

$$
\left|\partial_{x_{n}}^{p} \partial_{\xi}^{q}\left[\xi^{2 m-1} \psi_{j k}\left(x_{n}, \xi\right)\right]\right| \leq C_{p q}\langle\xi\rangle^{2 m-q}\left\langle x_{n}\right\rangle^{1-\theta-p}, \quad x_{n} \in \mathbb{R}, \xi \in \mathbb{R}^{*} .
$$

2) One has supp $r_{j k} \subset \operatorname{supp} \gamma_{j k}$, and for every $p, q \in \mathbb{N}$, there exist constants $C_{p q}^{\prime}$ (depending also on $j$ and $k$ ) such that

$$
\left|\partial_{x_{n}}^{p} \partial_{\xi}^{q} r_{j k}\left(x_{n}, \xi\right)\right| \leq C_{p q}^{\prime}\left\langle x_{n}\right\rangle^{-1-\theta-p}, \quad x_{n} \in \mathbb{R}, \xi \in \mathbb{R}^{*} .
$$

3) One has the equality

$$
-\nabla \cdot\left(c_{j}+\delta^{L}\right) \nabla\left(e^{i \psi_{j k}} \Phi_{k} \gamma_{j k}\right)=e^{i \psi_{j k}} \Phi_{k}\left[\lambda_{j k}(\xi) \gamma_{j k}+r_{j k}\right], \quad x \in \Omega, \xi \in \mathbb{R}^{*} .
$$

Proof. Suppose $\psi_{j k}$ satisfying 1): a direct calculation gives easily that

$$
\begin{aligned}
(3.7)- & \nabla \cdot\left(c_{j}+\sigma^{L}\right) \nabla\left(e^{i \psi_{j k}} \Phi_{k} \gamma_{j k}\right) \\
& =e^{i \psi_{j k}} \Phi_{k}\left[\lambda_{j k}(\xi) \gamma_{j k}+\gamma_{j k} E_{j k}\left(\partial_{x_{n}} \psi_{j k}\right)+r_{j k}^{\prime}\right] \quad \text { for } x \in \Omega, \xi \in \mathbb{R}^{*},
\end{aligned}
$$


where $r_{j k}^{\prime}$ satisfies 2) and

$$
E_{j k}(t):=\left(c_{j}+\delta^{L}\right) t^{2}+2 \xi\left(c_{j}+\delta^{L}\right) t+\left(\lambda_{k}+\xi^{2}\right) \delta^{L}, \quad t \in \mathbb{R} .
$$

We determine $\psi_{j k}$ as an approximate solution of the eikonal equation $E_{j k}\left(\partial_{x_{n}} \psi_{j k}\right)=0$. We set

$$
A_{j k}\left(x_{n}, \xi\right):=\frac{\lambda_{k}+\xi^{2}}{\xi^{2}\left(c_{j}+\delta^{L}\left(x_{n}\right)\right)} \delta^{L}\left(x_{n}\right), \quad x_{n} \in \mathbb{R}, \xi \in \mathbb{R}^{*},
$$

and choose $R_{k}$ in (3.2) such that $\left|A_{j k}\right| \leq 1 / 2$ on $\operatorname{supp} \gamma_{j k}$. Then on this support of $\gamma_{j k}$, we have $E_{j k}\left(B_{j k}^{\prime}\right)=0$ where $B_{j k}^{\prime}:=\xi\left(\sqrt{1-A_{j k}}-1\right)$. Now, we define $B_{j k}: \mathbb{R} \times \mathbb{R}^{*} \rightarrow \mathbb{R}$ by

$$
B_{j k}:=\xi \sum_{s=1}^{m} \frac{1}{2}\left(\frac{1}{2}-1\right) \cdots\left(\frac{1}{2}-s+1\right) \frac{\left(-A_{j k}\right)^{s}}{s !},
$$

and $\psi_{j k}: \mathbb{R} \times \mathbb{R}^{*} \rightarrow \mathbb{R}$ by

$$
\psi_{j k}\left(x_{n}, \xi\right):=\int_{0}^{x_{n}} B_{j k}(t, \xi) d t .
$$

Using (1.2), (3.8), (3.9) and (3.10), we see that $\psi_{j k}$ satisfies 1). Now with Taylor's formula, using (1.2), (3.8), (3.9) and with the choice of $m$, we see that, if $D_{j k}:=B_{j k}-B_{j k}^{\prime}$ on $\operatorname{supp} \gamma_{j k}$, then $\gamma_{j k} D_{j k}$ satisfies inequalities of type (3.5). Finally we have $\partial_{x_{n}} \psi_{j k}=B_{j k}=B_{j k}^{\prime}+D_{j k}$ and then

$$
\gamma_{j k} E_{j k}\left(\partial_{x_{n}} \psi_{j k}\right)=\gamma_{j k} E_{j k}\left(B_{j k}^{\prime}\right)+\left(c_{j}+\delta^{L}\right) \gamma_{j k} D_{j k}\left(D_{j k}+2 B_{j k}^{\prime}+2 \xi\right) .
$$

To get (3.6), we use (3.7) and choose $r_{j k}:=r_{j k}^{\prime}+\gamma_{j k} D_{j k}\left(c_{j}+\delta^{L}\right)\left(D_{j k}+2 B_{j k}^{\prime}+\right.$ $2 \xi)$.

\section{$\S 4$. A Class of Fourier Integral Operators}

With the notations of Section 3 and for $j=1,2, k \geq 1$, we define the real-valued function $\varphi_{j k} \in C^{\infty}\left(\mathbb{R} \times \mathbb{R}^{*}\right)$ by $\varphi_{j k}\left(x_{n}, \xi\right):=x_{n} \xi+\varphi_{j k}\left(x_{n}, \xi\right)$ and the operator $F_{j k}$ by

$$
F_{j k} f\left(x_{n}\right):=(2 \pi)^{-1 / 2} \int_{\mathbb{R}} e^{i \varphi_{j k}\left(x_{n}, \xi\right)} \gamma_{j k}\left(x_{n}, \xi\right) \widehat{f}(\xi) d \xi, \quad f \in \mathcal{S}(\mathbb{R}), x_{n} \in \mathbb{R},
$$

with $\widehat{f}(\xi):=(2 \pi)^{-1 / 2} \int_{\mathbb{R}} e^{-i t \xi} f(t) d t$. 
Lemma 4.1. 1) $F_{j k}: \mathcal{S}(\mathbb{R}) \rightarrow \mathcal{S}(\mathbb{R})$ is continuous.

2) $F_{j k}$ has a unique extension as an operator of $\mathcal{B}\left(L^{2}(\mathbb{R})\right)$.

Proof. For 1), we use the inequalities (3.4).

For 2), we remark firstly that the formal adjoint of $F_{j k}$ satisfies the equality

$$
\widehat{\left(F_{j k}^{*} g\right)}(\xi)=(2 \pi)^{-1 / 2} \int_{\mathbb{R}} e^{-i \varphi_{j k}\left(y_{n}, \xi\right)} \gamma_{j k}\left(y_{n}, \xi\right) g\left(y_{n}\right) d y_{n}, \quad g \in \mathcal{S}(\mathbb{R}), \quad \xi \in \mathbb{R} .
$$

Then

$$
\left(F_{j k} F_{j k}^{*} g\right)\left(x_{n}\right)=(2 \pi)^{-1} \int_{\mathbb{R}} K_{j k}\left(x_{n}, y_{n}\right) g\left(y_{n}\right) d y_{n}, \quad g \in \mathcal{S}(\mathbb{R})
$$

where

$$
K_{j k}\left(x_{n}, y_{n}\right)=\int_{\mathbb{R}} e^{i\left[\varphi_{j k}\left(x_{n}, \xi\right)-\varphi_{j k}\left(y_{n}, \xi\right)\right]} \gamma_{j k}\left(x_{n}, \xi\right) \gamma_{j k}\left(y_{n}, \xi\right) d \xi, \quad x_{n}, y_{n} \in \mathbb{R} .
$$

We have

$$
\varphi_{j k}\left(x_{n}, \xi\right)-\varphi_{j k}\left(y_{n}, \xi\right)=\left(x_{n}-y_{n}\right)\left(\xi+\zeta_{j k}\left(x_{n}, y_{n}, \xi\right)\right)
$$

with

$$
\zeta_{j k}\left(x_{n}, y_{n}, \xi\right):=\int_{0}^{1}\left(\partial_{x_{n}} \psi_{j k}\right)\left(t x_{n}+(1-t) y_{n}\right) d t .
$$

If $\left(x_{n}, \xi\right)$ and $\left(y_{n}, \xi\right)$ are in $\operatorname{supp} \gamma_{j k}$, we have $\left|t x_{n}+(1-t) y_{n}\right|=t\left|x_{n}\right|+(1-$ $t)\left|y_{n}\right| \geq R_{k}$ for every $t \in(0,1)$. Then using (3.4), we find a constant $C>0$ such that $\left|\partial_{\xi} \zeta_{j k}\left(x_{n}, y_{n}, \xi\right)\right| \leq C R_{k}^{-\theta} \leq 1 / 2$ if $R_{k}$ is large enough. In the integral (4.4), we can do the change of variables $\eta=\xi+\zeta_{j k}\left(x_{n}, y_{n}, \xi\right)$, i.e. $\xi=\xi_{j k}\left(x_{n}, y_{n}, \eta\right)$, since the derivatives with respect to $\eta$ of $\xi_{j k}$ are bounded on the support of the integrand. After an integration by part, we get for every $p \in \mathbb{N}$

$$
\begin{aligned}
K_{j k}\left(x_{n}, y_{n}\right)= & \left\langle x_{n}-y_{n}\right\rangle^{-2 p} \int_{\mathbb{R}} e^{i\left(x_{n}-y_{n}\right) \eta}\left(1-\partial_{\eta}^{2}\right)^{p}\left[\gamma_{j k}\left(x_{n}, \xi_{j k}\left(x_{n}, y_{n}, \eta\right)\right)\right. \\
& \left.\times \gamma_{j k}\left(y_{n}, \xi_{j k}\left(x_{n}, y_{n}, \eta\right)\right)\left(1+\left(\partial_{\xi} \zeta_{j k}\right)\left(x_{n}, y_{n}, \xi_{j k}\left(x_{n}, y_{n}, \eta\right)\right)\right)^{-1}\right] d \eta
\end{aligned}
$$

Thus there exists a constant $C>0$ such that $\left|K_{j k}\left(x_{n}, y_{n}\right)\right| \leq C\left\langle x_{n}-y_{n}\right\rangle^{-2 p}$ for every $x_{n}, y_{n} \in \mathbb{R}$ and $p \in \mathbb{N}$. Now using the Schur lemma, we deduce that $F_{j k} F_{j k}^{*}$ has a unique extension as an operator of $\mathcal{B}\left(L^{2}(\mathbb{R})\right)$, so that $F_{j k}^{*}$ and $F_{j k}$ have the same property. 
Remark 4.1. In fact $F_{j k} \in \mathcal{B}\left(L^{2}(\mathbb{R}), \mathcal{H}^{s}(\mathbb{R})\right)$ for all $s \in \mathbb{R}$.

Lemma 4.2. The operator $L_{j k}:=\langle\cdot\rangle^{-(1+\theta) / 2}\left[\langle\cdot\rangle^{(i+\theta) / 2}, F_{j k}\right]$, well defined on $\mathcal{S}(\mathbb{R})$, has a unique extension as an operator of $\mathcal{B}\left(L^{2}(\mathbb{R})\right)$.

Proof. If $a\left(x_{n}\right):=\left\langle x_{n}\right\rangle^{(1+\theta) / 2}, b\left(x_{n}, y_{n}\right):=a\left(x_{n}\right)^{-1}\left[a\left(x_{n}\right)-a\left(y_{n}\right)\right]$ and $\rho_{j k}\left(x_{n}, y_{n}, \xi\right):=\left(x_{n}-y_{n}\right) \xi+\psi_{j k}\left(x_{n}, \xi\right)$, we have

$$
\left(L_{j k} f\right)\left(x_{n}\right)=(2 \pi)^{-1 / 2} \int_{\mathbb{R}} \widetilde{K_{j k}}\left(x_{n}, y_{n}\right) f\left(y_{n}\right) d y_{n}, f \in \mathcal{S}(\mathbb{R}), x_{n} \in \mathbb{R},
$$

where

$$
\widetilde{K_{j k}}\left(x_{n}, y_{n}\right):=\int_{\mathbb{R}} e^{i \rho_{j k}\left(x_{n}, y_{n}, \xi\right)} b\left(x_{n}, y_{n}\right) \gamma_{j k}\left(x_{n}, \xi\right) d \xi, x_{n}, y_{n} \in \mathbb{R} .
$$

If $\left|x_{n}-y_{n}\right|>(1 / 2)\left|x_{n}\right|$ and $q \in \mathbb{N}, q \geq 3 /(2 \theta)$, we have $\left|\widetilde{K_{j k}}\left(x_{n}, y_{n}\right)\right|=$ $\left\langle x_{n}-y_{n}\right\rangle^{-2 q} b\left(x_{n}, y_{n}\right) \int_{\mathbb{R}} e^{i\left(x_{n}-y_{n}\right) \xi}\left(1-\partial_{\xi}^{2}\right)^{q}\left[e^{i \psi_{j k}\left(x_{n}, \xi\right)} \gamma_{j k}\left(x_{n}, \xi\right)\right] d \xi$ and there exist constants $C_{j k}^{\prime}$ and $C_{j k}^{\prime \prime}$ such that

$$
\left|\widetilde{K_{j k}}\left(x_{n}, y_{n}\right)\right| \leq C_{j k}^{\prime}\left\langle x_{n}-y_{n}\right\rangle^{-2 q}\left|x_{n}-y_{n}\right|\left\langle x_{n}\right\rangle^{2 q(1-\theta)} \leq C_{j k}^{\prime \prime}\left\langle x_{n}-y_{n}\right\rangle^{-2} .
$$

If $\left|x_{n}-y_{n}\right| \leq(1 / 2)\left|x_{n}\right|$, then $(2 / 3)\left|y_{n}\right| \leq\left|x_{n}\right| \leq 2\left|y_{n}\right|$. For $p \in \mathbb{N}, p \geq(2-\theta) / \theta$, we write

$$
b\left(x_{n}, y_{n}\right)=s_{p}\left(x_{n}, y_{n}\right)+r_{p}\left(x_{n}, y_{n}\right)
$$

where

$$
s_{p}\left(x_{n}, y_{n}\right):=-a\left(x_{n}\right)^{-1} \sum_{t=1}^{p} \frac{1}{t !}\left(y_{n}-x_{n}\right)^{t} a^{(t)}\left(x_{n}\right)
$$

and

$$
r_{p}\left(x_{n}, y_{n}\right):=-a\left(x_{n}\right)^{-1} \frac{1}{p !}\left(y_{n}-x_{n}\right)^{p+1} \int_{0}^{1}(1-\tau)^{p} a^{(p+1)}\left(x_{n}+\tau\left(y_{n}-x_{n}\right)\right) d \tau .
$$

According to the decomposition (4.11), we write

$$
\widetilde{K_{j k}}\left(x_{n}, y_{n}\right):={\widetilde{K_{j k}}}^{\prime}\left(x_{n}, y_{n}\right)+{\widetilde{K_{j k}}}^{\prime \prime}\left(x_{n}, y_{n}\right) .
$$

On the one hand, the identity

$$
\left(y_{n}-x_{n}\right)^{p+1} e^{i \rho_{j k}\left(x_{n}, y_{n}, \xi\right)}=\left[\left(-D_{\xi}\right)^{p+1} e^{i\left(x_{n}-y_{n}\right) \xi}\right] e^{i \psi_{j k}\left(x_{n}, \xi\right)}
$$


and integrations by part give a constant $C_{p, j, k}$ such that, for $\left|x_{n}-y_{n}\right| \leq$ $(1 / 2)\left|x_{n}\right|$,

$$
\left|{\widetilde{K_{j k}}}^{\prime \prime}\left(x_{n}, y_{n}\right)\right| \leq C_{p, j, k}\left\langle x_{n}\right\rangle^{-(p+1) \theta} \leq C_{p, j, k}\left\langle x_{n}-y_{n}\right\rangle^{-2}
$$

On the other hand, with similar integrations by parts, we see that the kernel ${\widetilde{K_{j k}}}^{\prime}\left(x_{n}, y_{n}\right)$ verifies an inequality similar to (4.10) for $\left|x_{n}-y_{n}\right| \geq(1 / 2)\left|x_{n}\right|$. Thus for every $x_{n}, y_{n} \in \mathbb{R}$, there exists a constant $C_{j k}$ such that $\left|{\widetilde{K_{j k}}}^{\prime \prime}\left(x_{n}, y_{n}\right)\right|$ $\leq C_{j, k}\left\langle x_{n}-y_{n}\right\rangle^{-2}$, so that the operator $T_{j k}^{\prime \prime}$ with the integral kernel $(2 \pi)^{-1 / 2}$ $\widetilde{K}_{j k}^{\prime \prime}$ is bounded on $L^{2}(\mathbb{R})$.

Finally the operator $T_{j k}^{\prime}$ with the integral kernel $(2 \pi)^{-1 / 2}{\widetilde{K_{j k}}}^{\prime}$ is written as

$$
\left(T_{j k}^{\prime} f\right)\left(x_{n}\right)=\int_{\mathbb{R}} e^{i \varphi_{j k}\left(x_{n}, \xi\right)} e_{j k}\left(x_{n}, \xi\right) \widehat{f}(\xi) d \xi, \quad f \in \mathcal{S}(\mathbb{R}), x_{n} \in \mathbb{R},
$$

where $e_{j k} \in C^{\infty}(\mathbb{R} \times \mathbb{R})$, supp $e_{j k} \subset \operatorname{supp} \gamma_{j k}$ and all the derivatives of $e_{j k}$ are bounded. Using the proof of Lemma 4.1 , we get $T_{j k}^{\prime} \in \mathcal{B}\left(L^{2}(\mathbb{R})\right)$. The proof is complete since $L_{j k}=T_{j k}^{\prime}+T_{j k}^{\prime \prime}$ according to (4.8) and (4.14).

\section{$\S 5 . \quad$ Identification Operators}

With the notations of the previous sections, we consider the operators $G_{j}=G_{j}(\Lambda), j=1,2$, defined for $f \in C_{0}^{\infty}(\Omega)$ and $x \in \Omega$ by

$$
\left(G_{j} f\right)(x):=\sum_{k \geq 1} \int_{\mathbb{R}} e^{i \psi_{j k}\left(x_{n}, \xi\right)} \Phi_{k}(x, \xi) \gamma_{j k}\left(x_{n}, \xi\right) \widehat{f}_{k}(\xi) d \xi .
$$

Remark 5.1. The sum in (5.1) is finite since $\gamma_{j k}=0$ if $k \geq k_{j}(\Lambda)+1$.

Lemma 5.1. 1) $G_{j}$ has a unique extension as an operator of $\mathcal{B}\left(L^{2}(\Omega)\right.$, $\left.\mathcal{H}_{0}^{1}(\Omega)\right)$.

2) For all $f \in L^{2}(\Omega), G_{j} f$ is in $C^{\infty}(\Omega)$.

Proof. It is sufficient to take into account the following expression of $G_{j}$ :

$$
\left(G_{j} f\right)(x)=\sum_{k \geq 1} V_{k}\left(x^{\prime}\right)\left(F_{j k} f_{k}\right)\left(x_{n}\right), \quad f \in C_{0}^{\infty}(\Omega),
$$

where $f_{k}$ is defined by (2.4) and to use Remark 4.1 and the fact that $V_{k} \in$ $\mathcal{H}_{0}^{1}\left(\Omega^{\prime}\right) \cap C^{\infty}\left(\Omega^{\prime}\right)$.

Remark now that for a fixed interval $\Lambda$ and for $1 \leq k \leq k_{j}(\Lambda), j=1,2$, we can choose $R_{k}$ in (3.2) independent of $k$, and consequently $\alpha_{j k}=: \alpha_{j}$. 
Proposition 5.1. The following relation holds:

$$
\sum_{j=1,2}\left[\alpha_{j}^{2} \beta^{2}\left(H_{j}\right)-G_{j} G_{j}^{*}\right] \in \mathcal{K}\left(L^{2}(\Omega)\right)
$$

Proof. Using (2.5) and (2.8), we get

$$
\begin{aligned}
& \sum_{j=1,2}\left[\alpha_{j}^{2} \beta^{2}\left(H_{j}\right) f\right](x) \\
& \quad=(2 \pi)^{-1 / 2} \sum_{j=1,2} \sum_{k \geq 1} V_{k}\left(x^{\prime}\right) \int_{\mathbb{R}} e^{i x_{n} \xi} \alpha_{j}^{2}\left(x_{n}\right) \beta^{2}\left(\lambda_{j k}(\xi)\right) \widehat{f}_{k}(\xi) d \xi
\end{aligned}
$$

for $f \in C_{0}^{\infty}(\Omega), x \in \Omega$. On the other hand, the formal adjoint of $G_{j}$ satisfies

$$
\left(G_{j}^{*} g\right)_{k}=F_{j k}^{*} g_{k}, g \in C_{0}^{\infty}(\Omega), \quad j=1,2, k \geq 1 .
$$

Then we have

$$
\left(G_{j} G_{j}^{*} f\right)(x)=(2 \pi)^{-1} \sum_{k \geq 1} V_{k}\left(x^{\prime}\right) \int_{\mathbb{R}} K_{j k}\left(x_{n}, y_{n}\right) f_{k}\left(y_{n}\right) d y_{n}, \quad f \in C_{0}^{\infty}(\Omega),
$$

where $K_{j k}$ is defined by (4.4) or(4.7). Using the identities

$$
\begin{aligned}
\alpha_{j}\left(y_{n}\right)-\alpha_{j}\left(x_{n}\right) & =\left(y_{n}-x_{n}\right) \int_{0}^{1} \alpha_{j}^{\prime}\left(x_{n}+t\left(y_{n}-x_{n}\right)\right) d t, \\
1-\left(1+\partial_{\xi} \zeta_{j k}\right)^{-1} & =\left(\partial_{\xi} \zeta_{j k}\right)\left(1+\partial_{\xi} \zeta_{j k}\right)^{-1}, \quad \eta-\xi_{j k}=\zeta_{j k}\left(x_{n}, y_{n}, \xi_{j k}\right),
\end{aligned}
$$

(4.6) and the inequalities (3.4), it is easily seen that $K_{j k}=K_{j k}^{\prime}+K_{j k}^{\prime \prime}$ with

$$
K_{j k}^{\prime}\left(x_{n}, y_{n}\right)=\int_{\mathbb{R}} e^{i\left(x_{n}-y_{n}\right) \eta} \gamma_{j k}^{2}\left(x_{n}, \eta\right) d \eta, \quad x_{n}, y_{n} \in \mathbb{R},
$$

while, for every $p, q \in \mathbb{N}$, there exists a constant $C_{p q}$ (depending also on $j$ and $k$ ) such that

$$
\left|\partial_{x_{n}}^{q} K_{j k}^{\prime \prime}\left(x_{n}, y_{n}\right)\right| \leq C_{p q}\left\langle x_{n}\right\rangle^{-\theta}\left\langle x_{n}-y_{n}\right\rangle^{-2 p}, \quad x_{n}, y_{n} \in \mathbb{R}
$$

Then the operator $T_{j}$ defined by

$$
\left(T_{j} f\right)(x):=(2 \pi)^{-1} \sum_{k \geq 1} V_{k}\left(x^{\prime}\right) \int_{\mathbb{R}} K_{j k}^{\prime \prime}\left(x_{n}, y_{n}\right) f_{k}\left(y_{n}\right) d y_{n}, \quad f \in C_{0}^{\infty}(\Omega),
$$

is such that $\langle\cdot\rangle^{\theta} T_{j} \in \mathcal{B}\left(L^{2}(\Omega), \mathcal{H}_{0}^{1}(\Omega)\right)$, so that $T_{j} \in \mathcal{K}\left(L^{2}(\Omega)\right), j=1,2$.

Finally, comparing the relations (5.4), (5.6) and (5.7), the operator on the left-hand side of (5.3) is equal to $T_{1}+T_{2}$, and the proof is ended. 


\section{§6. Existence of the Generalized Wave Operators}

We use the following result of existence for the wave operators.

Proposition 6.1. Let $\mathcal{J}$ be an open set in $\mathbb{R}, \mathcal{H}$ be a separable complex Hilbert space, $T_{1}$ and $T_{2}$ be two self-adjoint operators in $\mathcal{H}, S$ be an operator in $\mathcal{B}(\mathcal{H}), A_{k}$ and $B_{k}, k=1, \ldots, N$, be operators with dense domains in $\mathcal{H}$. Suppose that

1) $\mathcal{J}=\bigcup_{i \in \mathbb{N}^{*}} \mathcal{J}_{i}$ where each $\mathcal{J}_{i}$ is a bounded open interval, and $\mathcal{J}_{i} \cap \mathcal{J}_{k}=\emptyset$ if $i \neq k$.

2) $A_{k}$ is $T_{1}$-bounded and locally $T_{1}$-smooth on $\mathcal{J}_{i}$, for $1 \leq k \leq N, i \geq 1$.

3) $B_{k}$ is $T_{2}$-bounded and locally $T_{2}$-smooth on $\mathcal{J}_{i}$, for $1 \leq k \leq N, i \geq 1$.

4) $T_{2} S-S T_{1}=\sum_{k=1}^{N} B_{k}^{*} A_{k}$ holds in the sense of forms, that is

$$
\left(T_{2} u, S v\right)_{\mathcal{H}}-\left(u, S T_{1} v\right)_{\mathcal{H}}=\sum_{1 \leq k \leq N}\left(B_{k} u, A_{k} v\right)_{\mathcal{H}}, \quad u \in D\left(T_{2}\right), v \in D\left(T_{1}\right) .
$$

5) Both sets $\sigma\left(T_{1}\right) \backslash \mathcal{J}$ and $\sigma\left(T_{2}\right) \backslash \mathcal{J}$ have Lebesgue measure 0 .

Then the generalized wave operators $s-\lim _{t \rightarrow \pm \infty} e^{i t T_{2}} S e^{-i t T_{1}} P_{a c}\left(T_{1}\right)$ and $s-\lim _{t \rightarrow \pm \infty} e^{i t T_{1}} S^{*} e^{-i t T_{2}} P_{a c}\left(T_{2}\right)$ exist.

The proof of this proposition is in [12] for $S=I d_{\mathcal{H}}$ and in [16] for the general case $S$ in $\mathcal{B}(\mathcal{H})$.

Remark 6.1 (see [12] and [1]). If $T$ is a self-adjoint operator on $\mathcal{H}$ and $A$ is a $T$-bounded operator, in order to verify that $A$ is locally $T$-smooth on an open interval $I \subset \mathbb{R}$, it is suffcient to verify, for every compact set $K \subset I$,

$$
\sup _{\lambda \in K, 0<\epsilon<1}\left\|A(T-\lambda-i \epsilon)^{-1} A^{*}\right\|_{\mathcal{B}(\mathcal{H})}<+\infty .
$$

Theorem 6.1. With the assumptions (i) and (ii) of the introduction and with $G_{j}$ defined by (5.1), there exist the following generalized wave operators:

$$
\begin{aligned}
\Omega_{j}^{ \pm}(\Lambda) & :=s-\lim _{t \rightarrow \pm \infty} e^{i t H} G_{j} e^{-i t H_{j}}, \quad j=1,2, \\
W_{j}^{ \pm}(\Lambda) & :=s-\lim _{t \rightarrow \pm \infty} e^{i t H_{j}} G_{j}^{*} e^{-i t H} P_{a c}(H), \quad j=1,2 .
\end{aligned}
$$

Proof. We apply Proposition 6.1 with $T_{1}=H_{j}, T_{2}=H, \mathcal{J}=\mathbb{R} \backslash(\tau(H) \cup$ $\left.\sigma_{p}(H)\right), S=G_{j}(\Lambda), \mathcal{H}=L^{2}(\Omega)$. The assumptions 1) and 5) are fullfilled. Using point 4) of Proposition 2.1 and (5.1), we have, for $v \in D\left(H_{j}\right), j=1,2, x \in \Omega$,

$$
G_{j} H_{j} v(x)=\sum_{k \geq 1} \int_{\mathbb{R}} e^{i \psi_{j k}\left(x_{n}, \xi\right)} \Phi_{k}(x, \xi) \gamma_{j k}\left(x_{n}, \xi\right) \lambda_{j k}(\xi) \widehat{v_{k}}(\xi) d \xi .
$$


On the other hand, if $u \in D(H), v \in D\left(H_{j}\right)$ and if we take into account the support of $\gamma_{j k}$, we have

$$
\left(H u, G_{j} v\right)_{L^{2}(\Omega)}=\int_{\Omega}\left(c_{j}+\delta^{L}\right) \nabla u \cdot \nabla G_{j} v d x+\int_{\Omega} \delta^{S} \nabla u \cdot \nabla G_{j} v d x .
$$

With (3.6) and (6.4), we see that the first integral on the right-hand side of (6.5) with $u \in C_{0}^{\infty}(\Omega)$ is equal to

$$
-\int_{\Omega} u \nabla \cdot\left(c_{j}+\delta^{L}\right) \nabla G_{j} v d x=\left(u, G_{j} H_{j} v\right)_{L^{2}(\Omega)}+\left(u, \widetilde{G_{j}} v\right)_{L^{2}(\Omega)},
$$

where

$$
\widetilde{G_{j}} v(x):=\sum_{k \geq 1} \int_{\mathbb{R}} e^{i \psi_{j k}\left(x_{n}, \xi\right)} \Phi_{k}(x, \xi) r_{j k}\left(x_{n}, \xi\right) \widehat{v_{k}}(\xi) d \xi, \quad j=1,2, x \in \Omega .
$$

Then the assumption 4) will be satisfied with $N=n+1$,

$$
\begin{aligned}
B_{k} u & :=\left\langle x_{n}\right\rangle^{1+\theta} \delta^{S}\left\langle x_{n}\right\rangle^{-(1+\theta) / 2} \partial_{x_{k}} u, A_{k} v:=\left\langle x_{n}\right\rangle^{-(1+\theta) / 2} \partial_{x_{k}} G_{j} v \text { if } 1 \leq k \leq n, \\
B_{n+1} u & :=\left\langle x_{n}\right\rangle^{-(1+\theta) / 2} u \text { and } \quad A_{n+1} v:=\left\langle x_{n}\right\rangle^{(1+\theta) / 2} \widetilde{G}_{j} v .
\end{aligned}
$$

Using (2.10) with $t=(1+\theta) / 2$ and (6.1), we see that the operators $B_{k}, 1 \leq$ $k \leq N$, satisfy the assumption 3$)$. We write now the identity $\left\langle x_{n}\right\rangle^{-t} G_{j} v=$ $\left\langle x_{n}\right\rangle^{-t}\left[G_{j},\left\langle x_{n}\right\rangle^{t}\right]\left\langle x_{n}\right\rangle^{-t} v+G_{j}\left\langle x_{n}\right\rangle^{-t} v$. Lemma 4.2 and the relation (5.2) imply that $A_{k}, 1 \leq k \leq n$, satisfy the assumption 2). Finally, with the inequalities

(3.5), we see that the operator $\left\langle x_{n}\right\rangle^{1+\theta} \widetilde{G_{j}}$ has the properties of $G_{j}$. In order to verify the assumption 2) for $A_{n+1}$, it is now sufficient to remark the identity

$$
\left\langle x_{n}\right\rangle^{t} \widetilde{G_{j}} v=\left(\left\langle x_{n}\right\rangle^{-t}\left[\left\langle x_{n}\right\rangle^{2 t} \widetilde{G_{j}},\left\langle x_{n}\right\rangle^{t}\right]\left\langle x_{n}\right\rangle^{-t}+\left\langle x_{n}\right\rangle^{2 t} \widetilde{G_{j}}\left\langle x_{n}\right\rangle^{-t}\right) v .
$$

\section{$\S 7 . \quad$ Asymptotic Completeness}

Remark 7.1. The following statements follow directly from the general properties of the generalized wave operators.

a) $\left(\Omega_{j}^{ \pm}(\Lambda)\right)^{*}=W_{j}^{ \pm}(\Lambda), \quad j=1,2$.

b) $\operatorname{Ran} \Omega_{1}^{ \pm}(\Lambda) \perp \operatorname{Ran} \Omega_{2}^{ \pm}(\Lambda)$.

c) If $E(\cdot)$ (respectively $E_{j}(\cdot)$ ) is the spectral measure of $H$ (respectively $H_{j}$ ), one has, for every Borel set $\mathcal{J} \subset \mathbb{R}$,

$$
E(\mathcal{J}) \Omega_{j}^{ \pm}(\Lambda)=\Omega_{j}^{ \pm}(\Lambda) E_{j}(\mathcal{J}), \quad j=1,2 .
$$

d) $\operatorname{Ran} \Omega_{j}^{ \pm}(\Lambda) \subset \mathcal{H}_{a c}(H), \quad j=1,2$. 
Theorem 7.1. Under the assumptions (i) and (ii) of the introduction, one has the following property of asymptotic completeness:

$$
P_{a c}(H)=\sum_{j=1,2} \Omega_{j}^{ \pm}(\Lambda) W_{j}^{ \pm}(\Lambda) \text { on } E(\Lambda) \mathcal{H} .
$$

Proof. It is sufficient to prove that if $f \in E(\Lambda) \mathcal{H}$ (in particular $f \in$ $\left.\mathcal{H}_{a c}(H)\right)$, and $f_{j}^{ \pm}:=W_{j}^{ \pm}(\Lambda) f, j=1,2$, then the following equality holds:

$$
\lim _{t \rightarrow \pm \infty}\left\|e^{-i t H} f-\sum_{j=1,2} G_{j} e^{-i t H_{j}} f_{j}^{ \pm}\right\|_{L^{2}(\Omega)}=0 .
$$

From $\lim _{t \rightarrow \pm \infty}\left\|G_{j}^{*} e^{-i t H} f-e^{-i t H_{j}} f_{j}^{ \pm}\right\|_{L^{2}(\Omega)}=0, j=1,2$, we get

$$
\lim _{t \rightarrow \pm \infty}\left\|G_{j} G_{j}^{*} e^{-i t H} f-G_{j} e^{-i t H_{j}} f_{j}^{ \pm}\right\|_{L^{2}(\Omega)}=0, \quad j=1,2 .
$$

Using Lemma 2.1, we see that the operator $K_{1}:=\beta^{2}(H)-\sum_{j=1,2} \alpha_{j}^{2} \beta^{2}\left(H_{j}\right)$ is in $\mathcal{K}\left(L^{2}(\Omega)\right)$. From Proposition 5.1, the operator $K_{2}:=\sum_{j=1,2}\left[\alpha_{j}^{2} \beta^{2}\left(H_{j}\right)-\right.$ $\left.G_{j} G_{j}^{*}\right]$ is also in $\mathcal{K}\left(L^{2}(\Omega)\right)$. Since $\lim _{t \rightarrow \pm \infty} e^{-i t H} f=0$ for the weak topology of $L^{2}(\Omega)$, we get $\lim _{t \rightarrow \pm \infty}\left\|\left(K_{1}+K_{2}\right) e^{-i t H} f\right\|=0$. Finally, the relation (7.4) and the equality $\beta^{2}(H) e^{-i t H} f=e^{-i t H} f$ imply (7.3).

\section{§8. Free Modified Evolutions}

We use the notations of the Sections 3 to 5 , and denote by $\chi_{j}: \Omega \rightarrow \mathbb{R}$ the characteristic function of $\left\{x_{n} \in \mathbb{R} /(-1)^{j} x_{n}>0\right\}, j=1,2$.

Proposition 8.1. There exist real-valued functions $a_{j k}, b_{j k} \in C^{\infty}(\mathbb{R} \times$ $\left.\mathbb{R}^{*}\right), j=1,2, k \geq 1$, uniquely determined by the functions $\psi_{j k}$ of Proposition 3.1, such that

1) The following identity holds for $x_{n} \in \mathbb{R}$ and $\pm t>0$ :

$$
a_{j k}\left(x_{n}, t\right)=\mp \frac{\pi}{4}+\frac{x_{n}^{2}}{4 c_{j}^{2} t}\left(2 c_{j}-1\right)-c_{j} \lambda_{k} t+b_{j k}\left(x_{n}, t\right) .
$$

2) $b_{j k}$ is zero if $\psi_{j k}=0$.

3) The following relations hold for $j=1,2$ and $f \in L^{2}(\Omega)$ :

$$
\lim _{t \rightarrow \pm \infty}\left\|G_{j}(\Lambda) e^{-i t H_{j}} f-U_{j}(t) \beta\left(H_{j}\right) f\right\|_{L^{2}(\Omega)}=0,
$$

where $U_{j}(t), j=1,2, t \in \mathbb{R}^{*}$, are the operators of $\mathcal{B}\left(L^{2}(\Omega)\right)$ defined by

$$
\left[U_{j}(t) f\right](t):=\chi_{j}\left(x_{n}\right)\left|2 c_{j} t\right|^{-1 / 2} \sum_{k \geq 1} V_{k}\left(x^{\prime}\right) e^{i a_{j k}\left(x_{n}, t\right)} \widehat{f}_{k}\left(\frac{x_{n}}{2 c_{j} t}\right), \quad f \in L^{2}(\Omega) .
$$


Proof. It is clear that $U_{j}(t) \in \mathcal{B}\left(L^{2}(\Omega)\right)$ since, for $j=1,2, \pm t>0$ and $f \in L^{2}(\Omega)$,

$$
\left\|U_{j}(t) f\right\|^{2}=\sum_{k \geq 1} \int_{\mathbb{R}} \chi_{j}\left( \pm x_{n}\right)\left|\widehat{f_{k}}\left(x_{n}\right)\right|^{2} d x_{n} \leq\|f\|_{L^{2}(\Omega)}^{2} .
$$

We fix $f \in C_{0}^{\infty}(\Omega)$. Pointing out that $\lim _{t \rightarrow \pm \infty} e^{-i t H_{j}} f=0$ for the weak topology of $L^{2}(\Omega)$ and that $\chi_{j}-\alpha_{j k}, j=1,2, k \geq 1$, is with a compact support in $\mathbb{R}$, it is sufficient to prove $(8.2)$ with $\widetilde{G_{j}}(\Lambda)$ instead of $G_{j}(\Lambda)$ (where $\widetilde{G_{j}}(\Lambda)$ is obtained from $G_{j}(\Lambda)$ by substituting $\chi_{j}$ for $\left.\alpha_{j k}\right)$ since $G_{j}-\widetilde{G_{j}} \in \mathcal{K}\left(L^{2}(\Omega)\right)$. Using (2.8) and (5.2), we write

$$
\widetilde{G_{j}}(\Lambda) e^{-i t H_{j}} f(x)=\sum_{k \geq 1} V_{k}\left(x^{\prime}\right)\left(\widetilde{F_{j k}} f_{k}\right)\left(x_{n}\right), \quad x \in \Omega, j=1,2,
$$

where

$$
\left(\widetilde{F_{j k}} g\right)\left(x_{n}\right):=(2 \pi)^{-1 / 2} \int_{\mathbb{R}} e^{i t \Phi_{j k}\left(x_{n}, \xi, t\right)} \widetilde{\gamma_{j k}}\left(x_{n}, \xi\right) \widehat{g}(\xi) d \xi, \quad g \in C_{0}^{\infty}(\mathbb{R}),
$$

with

$$
\begin{aligned}
\widetilde{\gamma_{j k}}\left(x_{n}, \xi\right) & :=\chi_{j}\left(x_{n}\right) \beta_{j k}(\xi) \text { and } \\
\Phi_{j k}\left(x_{n}, \xi, t\right) & :=x_{n} t^{-1} \xi-c_{j}\left(\lambda_{k}+\xi^{2}\right)+t^{-1} \psi_{j k}\left(x_{n}, \xi\right), \quad x_{n} \in \mathbb{R}, \xi, t \in \mathbb{R}^{*} .
\end{aligned}
$$

We fix $\varepsilon>0$ small enough and $\rho \in C_{0}^{\infty}(\mathbb{R})$ an even real-valued function, such that $\rho(s)=1$ if $|s| \leq \varepsilon / 2, \rho(s)=0$ if $|s| \geq \varepsilon$. We set $\rho_{j k}^{\prime}\left(x_{n}, \xi, t\right):=$ $\rho\left(\left(\partial_{\xi} \Phi_{j k}\right)\left(x_{n}, \xi, t\right)\right)$ and $\rho_{j k}^{\prime \prime}:=1-\rho_{j k}^{\prime}$. According to $1=\rho_{j k}^{\prime}+\rho_{j k}^{\prime \prime}$, we write $\widetilde{F_{j k}}=\widetilde{F_{j k}}+\widetilde{F_{j k}}{ }^{\prime \prime}$. We set also

$$
A_{j k}:=\left\{\left(x_{n}, \xi, t\right) \in \operatorname{supp} \rho_{j k}^{\prime} / \operatorname{dist}\left(\xi, \operatorname{supp} \beta_{j k}\right)=d / 2,|t| \geq T\right\}
$$

where $d:=\operatorname{dist}\left(0, \operatorname{supp} \beta_{j k}\right)$ and $T \geq 1$ chosen large enough. We have

$$
\left(\partial_{\xi} \Phi_{j k}\right)\left(x_{n}, \xi, t\right)=\frac{x_{n}}{t}-2 c_{j} \xi+\frac{1}{t}\left(\partial_{\xi} \psi_{j k}\right)\left(x_{n}, \xi\right) .
$$

Taking (3.4) into account, we can find two constants $m$ and $M, 0<m<M$, such that if $T$ is large enough, the following estimates hold on $A_{j k}$ :

$$
m \leq\left|\frac{x_{n}}{2 c_{j} t}\right| \leq M \text { and }\left|\frac{x_{n}}{2 c_{j} t}-\xi\right| \leq \frac{\varepsilon}{4 c_{j}} .
$$


We can assume $\varepsilon /\left(4 c_{j}\right) \leq d / 4$. We have also

$$
\left(\partial_{\xi}^{2} \Phi_{j k}\right)\left(x_{n}, \xi, t\right)=-2 c_{j}+t^{-1}\left(\partial_{\xi}^{2} \psi_{j k}\right)\left(x_{n}, \xi\right)
$$

so that $\left|\partial_{\xi}^{2} \Phi_{j k}\right| \geq c_{j}$ on $A_{j k}$ if $T$ is large enough. We see that for $T$ large enough, for $x_{n}, t \in \mathbb{R},|t| \geq T$, such that dist $\left(x_{n} /\left(2 c_{j} t\right), \operatorname{supp} \beta_{j k}\right) \leq(d / 4)$, there exists a unique solution $\xi_{j}=\xi_{j k}\left(x_{n}, t\right)$ of the equation $\left(\partial_{\xi} \Phi_{j k}\right)\left(x_{n}, \xi_{j}, t\right)=0$ such that $\left|x_{n} /\left(2 c_{j} t\right)-\xi_{j}\right| \leq \varepsilon /\left(4 c_{j}\right)$ and thus $\left(x_{n}, \xi_{j}, t\right) \in A_{j k}$. Using (8.8) and (3.4), we get that the derivatives of $\Phi_{j k}$ are bounded on $A_{j k}$ and then, with Theorem 7.7.6 of [8], we have

$$
\begin{gathered}
\left(\widetilde{F_{j k}} g\right)\left(x_{n}\right) \sim e^{i t \Phi_{j k}\left(x_{n}, \xi_{j k}\left(x_{n}, t\right), t\right)}\left|\left(\partial_{\xi}^{2} \psi_{j k}\right)\left(x_{n}, \xi_{j k}\left(x_{n}, t\right)\right)-2 c_{j} t\right|^{-1 / 2} \\
e^{\frac{\pi i}{4} \operatorname{sgn}\left[\left(\partial_{\xi}^{2} \psi_{j k}\right)\left(x_{n}, \xi_{j k}\left(x_{n}, t\right)\right)-2 c_{j} t\right]} \widetilde{\gamma_{j k}}\left(x_{n}, \xi_{j k}\left(x_{n}, t\right)\right) \widehat{g}\left(\xi_{j k}\left(x_{n}, t\right)\right)
\end{gathered}
$$

modulo terms the $L^{2}(\mathbb{R})$-norms of which go to zero if $|t| \rightarrow \infty$. Thanks to the inequalities (3.4) and (8.8), for every $s \in \mathbb{N}$, there exists a positive constant $C_{s}$ such that $\left|\left(\partial_{\xi}^{s} \psi_{j k}\right)\left(x_{n}, \xi_{j k}\left(x_{n}, t\right)\right)\right| \leq C_{s}|t|^{1-\theta},|t| \geq T$. In particular, using the equality

$$
\xi_{j k}\left(x_{n}, t\right)=\frac{x_{n}}{2 c_{j} t}+\frac{1}{2 c_{j} t}\left(\partial_{\xi} \psi_{j k}\right)\left(x_{n}, \xi_{j k}\left(x_{n}, t\right)\right),
$$

we get the inequality $\left|\xi_{j k}\left(x_{n}, t\right)-\left(x_{n} / 2 c_{j} t\right)\right| \leq\left(2 c_{j}\right)^{-1} C_{s}|t|^{-\theta},|t| \geq T$. On the other hand, to eliminate some terms (depending explicitely on $\xi_{j k}$ ) of $\Phi_{j k}$, we replace $\xi_{j k}$ by its expression coming from (8.10) and we use a Taylor's development of finite order to express $\left(\partial_{\xi}^{s} \psi_{j k}\right)\left(x_{n}, \xi_{j k}\left(x_{n}, t\right)\right)$ in function of the derivatives $\left(\partial_{\xi}^{r} \psi_{j k}\right)\left(x_{n},\left(x_{n} / 2 c_{j} t\right)\right)$, the powers of $\xi_{j k}\left(x_{n}, t\right)-\left(x_{n} / 2 c_{j} t\right)$ and a convenient remainder. Repeating several times these two operations, we find the functions $a_{j k}$ and $b_{j k}$ satisfying (8.1), independent of $\xi_{j k}\left(x_{n}, t\right)$ and such that $\left|t \Phi_{j k}\left(x_{n}, \xi_{j k}\left(x_{n}, t\right)\right)-a_{j k}\left(x_{n}, t\right)\right| \leq C|t|^{-\delta}$ holds on $A_{j k}$ with two positive constants $C$ and $\delta$. We deduce

$$
\left(\widetilde{F_{j k}} g\right)\left(x_{n}\right) \sim \chi_{j}\left(x_{n}\right)\left|2 c_{j} t\right|^{-1 / 2} e^{i a_{j k}\left(x_{n}, t\right)} \beta_{j k}\left(\frac{x_{n}}{2 c_{j} t}\right) \widehat{g}\left(\frac{x_{n}}{2 c_{j} t}\right) .
$$

To estimate $\widetilde{F_{j k}}$, we write it under the form

$$
\left(\widetilde{F_{j k}}{ }^{\prime \prime} g\right)\left(x_{n}\right)=-(2 \pi)^{-1 / 2} t^{-1} \int_{\mathbb{R}} e^{i t \Phi_{j k}\left(x_{n}, \xi\right)} D_{\xi}\left(\frac{\rho_{j k}^{\prime \prime}}{\partial_{\xi} \Phi_{j k}} \widetilde{\gamma_{j k}} \widehat{g}\right) d \xi
$$

In (8.8), we can assume $M$ large enough. If $\left|x_{n} /\left(2 c_{j} t\right)\right| \leq M,|t| \geq T, T$ large enough, we have also $\left\langle x_{n}\right\rangle / 2 c_{j}|t| \leq M+1$ and the derivatives of $\Phi_{j k}$ are 
bounded. Therefore there exists a constant $C>0$ such that for $\left|x_{n} /\left(2 c_{j} t\right)\right| \leq$ $M,|t| \geq T$, we have

$$
\left|\left(\widetilde{F_{j k}}{ }^{\prime \prime} g\right)\left(x_{n}\right)\right| \leq C|t|^{-1} \leq C\left(2 c_{j}(M+1)\right)^{3 / 4}|t|^{-1 / 4}\left\langle x_{n}\right\rangle^{-3 / 4} .
$$

If $\left|x_{n} /\left(2 c_{j} t\right)\right|>M$, the derivative of $\rho_{j k}^{\prime \prime}$ is zero for $\xi \in \operatorname{supp} \beta_{j k}$ and the only term with problems is $\left(\partial_{\xi}^{2} \Phi_{j k}\right)\left(\partial_{\xi} \Phi_{j k}\right)^{-2}$. But if $M$ and $T$ are large enough, there exist positive constants $c$ and $C$ such that $\left|\partial_{\xi}^{2} \Phi_{j k}\left(x_{n}, \xi\right)\right| \leq C(1+$ $\left.|t|^{-1}\left\langle x_{n}\right\rangle^{1-\theta}\right)$ and $\left|\partial_{\xi} \Phi_{j k}\left(x_{n}, \xi\right)\right| \geq c t^{-1}\left\langle x_{n}\right\rangle$. On the support of $\rho_{j k}^{\prime \prime}$, we have also $\left|\partial_{\xi} \Phi_{j k}\left(x_{n}, \xi\right)\right| \geq \varepsilon / 2$ and then we can find another constant $C_{0}$ such that for $\left|x_{n} /\left(2 c_{j} t\right)\right|>M,|t| \geq T$,

$$
\left.\mid \widetilde{F_{j k}}{ }^{\prime \prime} g\right)\left.\left(x_{n}\right)\left|\leq C_{0}\left\langle x_{n}\right\rangle^{-1} \leq C_{0}\left(2 c_{j} M\right)^{-1 / 4}\right| t\right|^{-1 / 4}\left\langle x_{n}\right\rangle^{-3 / 4} .
$$

From (8.13) and (8.14), we get

$$
\lim _{t \rightarrow \pm \infty}\left\|\widetilde{F_{j k}}{ }^{\prime \prime} g\right\|=0
$$

The relation (8.2) follows from (2.8), (8.3), (8.5), (8.11) and (8.15).

Proof of Theorem 1.1. Using Theorem 6.1 and (8.2), we deduce

$$
\Omega_{j}^{ \pm}(\Lambda)=s-\lim _{t \rightarrow \pm \infty} e^{i t H} U_{j}(t) \beta\left(H_{j}\right), \quad j=1,2,
$$

so that there exist the operators $\Omega_{j}^{ \pm}$defined by (1.7) and

$$
\Omega_{j}^{ \pm}(\Lambda)=\Omega_{j}^{ \pm} \beta\left(H_{j}\right), \quad j=1,2 .
$$

Property (1.8) of asymptotic completeness follows from (8.17) and Theorem (7.1).

Proof of Corollary 1.1. It is sufficient to use the relation (8.4) which implies

$$
\left\|\Omega_{j}^{ \pm} f\right\|_{L^{2}(\Omega)}^{2}=\sum_{k \geq 1} \int_{\mathbb{R}} \chi_{j}\left( \pm x_{n}\right)\left|\tilde{f}_{k}\left(x_{n}\right)\right|^{2} d x_{n}, \quad f \in L^{2}(\Omega) .
$$

\section{References}

[1] Amrein, W., Boutet de Monvel, A. and Georgescu, V., C $C_{0}$-groups, commutator methods and spectral theory of $N$-body Hamiltonians, Birkhäuser, 1996. 
[2] Boutet de Monvel, A., Georgescu, V. and Soffer, A., N-body Hamiltonians with hardcore interactions, Rev. Math. Phys., 6-4 (1994), 515-596.

[3] Boutet de Monvel, A. and Manda, D., Spectral and scattering theory for wave propagation in perturbed stratified media, J. Math. Anal. Appl., 191 (1995), 137-167.

[4] Croc, E. and Dermenjian, Y., Analyse spectrale d'une bande acoustique multistratifiée I, Principe d'absorption limite pour une stratification simple. SIAM J. Math. Anal., 26 (1995), 880-924.

[5] Croc, E. and Iftimie, V., Wave operators in a multistratified strip, Integr. Equ. Oper. Theory, 33 (1999), 389-405.

[6] DeBièvre, S. and Pravica, D. W., Spectral analysis for optical fibers and stratified fluids I., J. Funct. Anal., 98 (1991), 404-436.

[7] Dermenjian, Y., Durand, M. and Iftimie, V., Spectral Analysis of an acoustic multistratified perturbed cylinder, Comm. Partial Diff. Eq., 23 (1998), 141-169.

[8] Hörmander, L., The analysis of linear partial differential operators I, Springer-Verlag, 1983.

[9] Isozaki, H. and Kitada, H., Modified wave operators with time-independent modifiers, J. Fac. Sci. Univ. Tokyo, 32 (1985), 77-104.

[10] Kato, T., Perturbation theory for linear operators, Springer-Verlag, 1980.

[11] Mazja, V. G., Sobolev spaces, Springer-Verlag, 1985.

[12] Reed, M. and Simon, B., Methods of modern mathematical physics IV: Analysis of operators, Academic Press, 1978.

[13] Stein, E., Singular integrals and differentiability properties of functions, Princeton University Press, 1970.

[14] Weder, R., Spectral and scattering theory for wave propagation in perturbed stratified media, Springer-Verlag, 1991.

[15] Wilcox, C. H., Sound propagation in stratified fluids, Springer-Verlag, 1984.

[16] Yafaev, D. R., Mathematical scattering theory, Amer. Math. Soc., 1992.

[17] - The scattering amplitude for the Schrödinger equation with a long-range potential, Comm. Math. Phys., 191 (1998), 183-218. 